\title{
Zur Psychologie der Erderwärmung
}

\author{
Korrespondenzadresse \\ Prof. Dr. Dr. Manfred Spitzer \\ Universität Ulm \\ Abteilung für Psychiatrie \\ Leimgrubenweg 12-14 \\ $87054 \mathrm{Ulm}$
}

\author{
Bibliografie \\ DOI https://doi.org/10.1055/a-0976-0420 \\ Nervenheilkunde 2020; 39: 6-9 \\ (c) Georg Thieme Verlag KG Stuttgart · New York \\ ISSN 0722-1541
}

Meine beiden Beiträge in diesem Heft wurden zwischen Weihnachten und den Heiligen Dreikönigen verfasst - in gefühlten 100 Stunden und oft bei einem Kaminfeuer. Dies war nicht nur den Fenstern und der Zentralheizung (beides nicht mehr auf dem neuesten Stand) geschuldet, sondern auch dem Effekt der lodernden und knisternden Flammen auf mein Gefühl von Wohlbefinden in warmer Behaglichkeit - die Dänen nennen es Hygge [13]. „Alles bloß romantische Einbildung - und noch dazu der ganze Feinstaub und die Erderwärmung!“, werden jetzt manche entsetzt denken. Dass Feuer mehr ist und vor allem war als romantischer Firlefanz, habe ich im Beitrag „Geist und Gehirn“ beleuchtet [10].

Zur Problematik der Erderwärmung haben vor allem Klimaforscher, Meteorologen, Physiker, Biologen und andere Naturwissenschaftler Stellung bezogen. Bereits vor einigen Jahren meldeten sich auch Psychologen zu Wort, um aus ihrer Sicht die Probleme, die Menschen mit dem Klimawandel haben, zu beleuchten [11]. Sie identifizierten 5 klimarelevante psychologische Sachverhalte:

- Menschen stützen ihre Entscheidungen und ihr Verhalten allgemein eher auf persönliche Erfahrungen als auf komplexe abstrakte Sachverhalte. Dies stellt ein Problem dar, weil der Klimawandel sich aus statistischen Analysen ergibt und damit in Begriffen und Zahlen beschrieben wird, nicht in konkreten Erfahrungen. Mit der Zunahme extremer Wetterereignisse (Wirbelstürme, Überschwemmungen, Trockenheit) dringe der Klimawandel allerdings immer stärker in unsere Lebenswelt ein - wenn nicht als direkte Erfahrung, dann zumindest in den Medien. Man denke nur an die bildgewaltigen Buschbrände in Australien mit den apokalyptisch wirkenden Fotos und Videos.

- Aufgrund der enormen globalen Ausmaße des Problems des Klimawandels fühlen sich die Menschen machtlos. Statt zu verzweifeln (was nichts bringt) könne man aus psychologischer Sicht nur mit neuen sozialen Normen Abhilfe schaffen, die individuelles Handeln belohnen, auch wenn es sich anfühlt wie ein Tropfen auf dem heißen Stein.

- Menschen neigen dazu, in ihrem unmittelbaren persönlichen Umfeld anders zu handeln als gegenüber räumlich und zeitlich weiter entfernt liegenden und zudem unsicheren, d. h. nur mit gewissen Wahrscheinlichkeiten auftretenden Ereignissen. Das abstrakte Entfernte können Menschen leichter ignorieren als das unmittelbare Persönliche. Die Kommunikation über den Klimawandel könne wirksamer geschehen, wenn man sich stärker auf die regionalen Auswirkungen der Erwärmung konzentrierte.
- Die Einstellung von Menschen zu Risiken hängt von deren Framing ab, d. h. davon, ob sie als mögliche Gewinne oder Verluste dargestellt werden. Es hat sich in Untersuchungen gezeigt, dass Menschen beim Umgang mit Verlusten vergleichsweise risikobereiter sind. Daraus leiten die Autoren ab, dass man weniger über langfristige Verluste (infolge von Nichthandeln), sondern mehr über kurzfristigen Nutzen (Gewinn infolge von Handeln) sprechen sollte. Für solche „Sofortmaßnahmen“ erhielte man mehr öffentliche Unterstützung.

- Damit zusammenhängend sei zu beachten, dass Menschen eine prinzipielle Abneigung gegenüber Verboten haben und stattdessen eher auf beispielsweise finanzielle Anreize zur Energieeinsparung reagieren, insbesondere dann, wenn diese mit intrinsischen Motiven, anderen zu helfen und die Umwelt zu schützen, verknüpft werden. Da intrinsische Motivation (das Gute wollen) durch extrinsische Motivation (Geld) langfristig jedoch untergraben wird, sollten finanzielle Anreize vorsichtig dosiert und nur kurzfristig gegeben werden.

Die Autoren fassen ihre Sicht wie folgt zusammen. „Politiker sollten den Klimawandel nicht als fernes, künftiges, abstraktes Risiko eines großen Verlusts darstellen, sondern

- den Schwerpunkt ihrer Argumentation auf das persönliche Risiko im Hier und Jetzt legen,

- das persönliche, positiv erfahrungsgestützte Engagement emotional betonen,

- klimarelevante gesellschaftliche Gruppennormen unterstützen,

- auf die Vorteile von sofortigem Handeln setzen und

- an von jedem Menschen letztlich selbst akzeptierte, langfristige Ziele einer gesunden und menschengerechten Umwelt appellieren“ [11].

Im Sommer 2019 erschien ein Sammelband mit dem Titel „die kognitive Psychologie des Klimawandels“ [8], der 10 Beiträge enthält - von theoretischen Gesichtspunkten bis zur emotionalen Auswertung von Bildern des Klimawandels, die frei zur Verfügung gestellt werden. Es blieb nicht bei bloßen Veröffentlichungen. Im Herbst 2019 trafen 42 psychologische Fachgesellschaften auf internationaler Ebene zusammen (Kasten), um mit einer Stimme zur Lösung des Problems Klimawandel beizutragen. Am 15. November 2019 um 14.30 Uhr fanden unter der Schirmherrschaft der amerikanischen und portugiesischen psychologischen Gesellschaft (APA und OPP) in Lissabon das erste Treffen statt, der inaugural Interna- 


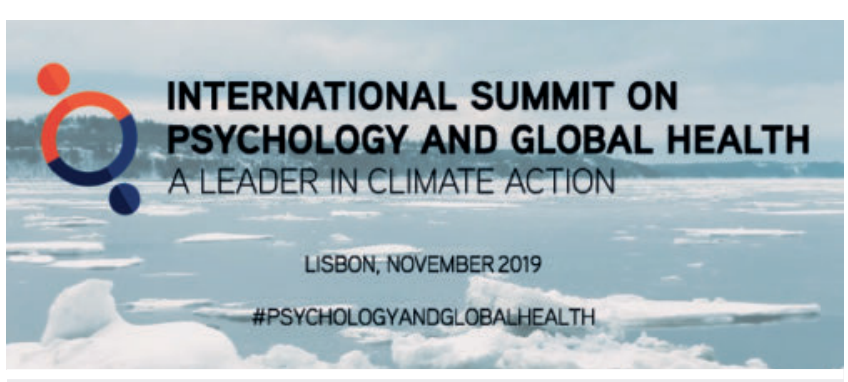

- Abb. 1 Screenshot der Webseite des International Summit on Psychology and Global Health in Lissabon [1].

tional Summit on Psychology and Global Health ( $\bullet$ Abb. 1). Dort wurde eine Erklärung verabschiedet (www.psychologyandglobalhealth.org/), der folgende Präambel vorausgeht:

\section{Psychologie und Klimamaßnahmen}

Unter den Klimawissenschaftlern besteht eine weitgehende Übereinstimmung dahingehend, dass der Klimawandel schneller voranschreitet als erwartet und zu einem großen Teil durch menschliches Verhalten verursacht wird. Neben der Bedrohung unserer natürlichen Umwelt und unserer körperlichen Gesundheit schädigt der Klimawandel auch die seelische Gesundheit und das Wohlbefinden der Menschen. Er kann das Gefühl der personalen und beruflichen Identität bedrohen, soziale Unterstützungssysteme schwächen und Gefühle von Hilflosigkeit, Angst und Fatalismus verstärken. Sowohl kurzfristige als auch langfristige Klimaveränderungen tragen nachweislich zum Verlust des sozialen Zusammenhalts bei und können sogar Feindseligkeiten und zwischenmenschliche Aggressionen sowie Konflikte zwischen Gruppen verstärken.

Die Wissenschaft der Psychologie kann nicht nur dabei helfen, umweltfreundliches Verhalten zu fördern, sondern kann auch den Menschen bei der Anpassung an den Klimawandel helfen. Psychologen können Resilienz aufbauen, Optimismus und die Bereitschaft zur Bewältigung fördern sowie soziale Bindungen stärken. Psychologen im Gesundheitswesen können zudem Menschen versorgen und betreuen, die an PTBS und anderen psychischen Störungen leiden, die im Zusammenhang mit dem Klimawandel stehen.

Kein einzelner Verband kann die globale Klimakrise wirksam bekämpfen. Die internationale Gemeinschaft der Psychologen muss sich daher zu einem Verband zusammenschließen, um unsere Mitglieder zu mobilisieren, unsere Stimme zu erheben und unsere Wissenschaft umzusetzen. Nur so können wir einen echten, dauerhaften gesellschaftlichen Wandel bewirken " [1].

Man sieht dem Text der Präambel an, dass es der wissenschaftlichen Psychologie nicht mehr um „kleine Tipps für Politiker“ geht, sondern um echtes soziales Engagement und um eine Reflexion darauf, wie wir mit dem Problem Klimawandel umgehen und was das wiederum mit uns macht. Ich möchte in aller Kürze - es wird ja fast schon zu viel darüber geredet - einige Gedanken aus wissenschaftlicher Sicht beitragen, die mir zu kurz gekommen scheinen.

\section{Angst versus Kreativität}

Als erstes macht der Gedanke Angst. Der Klimawandel ist nicht zu leugnen. Die vergangenen 5 Jahre waren die wärmsten seit es

\section{INTERNATIONAL SUMMIT ON PSYCHOLOGY} AND GLOBAL HEALTH

\section{Liste der teilnehmenden Fachgesellschaften}

Die gemeinsame Absichtserklärung einer besseren internationalen Zusammenarbeit auf dem Gebiet der Erderwärmung unterschrieben (www.psychologyandglobalhealth.org):

- American Psychological Association

- Order of Portuguese Psychologists

- Australian Psychological Society

- Bahamas Psychological Association

- Brazilian Society of Psychology

- British Psychological Society

- Canandian Psychological Association

- Caribbean Alliance of National Psychological Associations

- Chinese Psychological Society

- Asian Psychological Association

- College of Psychologists of Peru

- Colombian College of Psychologists

- Cuban Society of Health Psychology

- Cuban Society of Psychology

- European Federation of Psychologists‘ Associations

- Flemish Association for Clinical Psychologists

- German Psychological Society

- Guatemala College of Psychologists

- International Association of Applied Psychology

- International Council of Psychologists

- International Union of Psychological Science

- Jamaican Psychological Society

- Japanese Psychological Association

- Jordanian Clinical Psychologists Association

- Lebanese Psychological Association

- Mexican Psychological Society

- National Psychological Association of Ukraine

- New Zealand Psychological Society

- Nicaraguan Association for the Development of Psychology

- Nigerian Psychological Association

- Norwegian Psychological Association

- Order of Psychologists of Albania

- Pan-African Psychology Union

- Psychological Association of Namibia

- Psychological Society of Kenya

- Seoul National University

- Spanish Psychological Association

- Sri Lanka Psychological Association

- Swedish Psychological Association

- Uganda Council of Psychologists

- Uruguayan Psychological Association

- Zanzibar Professional Counselors Association

Wetteraufzeichnungen gibt. Extremes Wetter nimmt zu, d. h. es gibt mehr Trocken- und Dürreperioden und zugleich mehr Überschwemmungen durch tropische Stürme ${ }^{1}$, der Meeresspiegel

1 Allein in den USA betrugen die Kosten der 3 Hurrikans Katrina, Rita und Wilma etwa 130 Milliarden Dollar [3]. 
steigt, Ökosysteme werden zerstört, die Versorgung mit Wasser und Nahrungsmitteln gerät zunehmend in Gefahr [2]. Veränderungen von Wetter und Klima gab es immer, aber nicht in dieser Geschwindigkeit - Pflanzen, Tiere und Menschen haben keine Chance, sich anzupassen, wie das über Jahrzehntausende früher erfolgte.

Die Welt-Klimakonferenz in Madrid hat auf ganzer Linie versagt und damit gezeigt, dass die "Menschheit" weit davon entfernt ist, die Klimakatastrophe abzuwenden [12]. Der Mensch hat sich dank seiner kulturellen Leistungen wie nicht zuletzt des Feuers in den verschiedensten klimatischen Bedingungen eingerichtet, vom arktischen Sibirien bis zu heißen Steinwüsten - beides eigentlich vollkommen unbewohnbare Gegenden. Der Klimawandel bedeutet daher nicht, wie manchmal behauptet oder zumindest impliziert wird, das Ende allen Lebens auf der Erde oder den Untergang der menschlichen Zivilisation. Dennoch werden nach allem, was wir bisher wissen, die mangelnde und marode Infrastruktur, die zu schwachen politischen Entscheidungssysteme und vor allem Millionen Klimaflüchtlinge innerhalb weniger Jahrzehnte die Welt, wie wir sie jetzt kennen, in ein Chaos stürzen - oder so ähnlich, denken immer mehr Menschen.

Die Folge sind massive Ängste, die sich breit machen, wann immer es um den Klimawandel geht. Angst jedoch ist das größte Hemmnis von Kreativität. Wie die psychologische Forschung vielfach gezeigt hat, geht Angst mit Genauigkeit, „Kleinteiligkeit“ und Fehlervermeidung einher, nicht jedoch mit Offenheit, gedanklicher Weite und Mut für neue Ideen. Wer Angst hat, kann nicht kreativ sein, weil die Angst für Anspannung, Fokussierung und Kampf oder Flucht sorgt, nicht jedoch für das entspannte „Kommenlassen“ neuer Ideen. „Not macht erfinderisch“, wird dem oft entgegengehalten. Aber Not ist nicht das Gleiche wie Angst! Materielle Not führt durchaus zu neuen Problemlösungen, eben aus der Not heraus, weil sonst nichts mehr geht. Aber die kreative Idee zu einer solchen Lösung stellt sich nicht bei gleichzeitig vorhandener Angst ein, sondern auch in der Not nur dann, wenn keine akute Gefahr im Verzug ist, die Angst bereitet. Gerade dann, wenn man Kreativität braucht, um ein riesiges Problem zu lösen, führt die Angst, die uns das Problem macht, zu deren Hemmung. Das müssen wir zur Kenntnis nehmen und verhindern.

\section{Vorurteile versus Offenheit}

Angst bewirkt nicht nur weniger Kreativität, sondern auch weniger Offenheit und damit mehr Vorurteile. Im Hinblick auf das Problem der Erderwärmung ist von Bedeutung, dass ein Zusammenhang von existenziellen ökologischen Bedrohungen einerseits mit stärker vorhandenen Vorurteilen andererseits in einer Studie an 117157 Personen aus 25 Ländern gezeigt werden [4]. Die Daten stammten aus dem World Values Survey (WVS). Im Hinblick auf Vorurteile wurden die Leute gefragt, ob sie etwas dagegen hätten oder nicht, „wenn Sie in der Nähe z. B.

- Menschen einer anderen Rasse,

- Menschen einer anderen Religionszugehörigkeit,

- Immigranten,

- Menschen, die eine andere Sprache sprechen oder

- Menschen, die eine andere sexuelle Orientierung haben leben würden.

- unverheiratet zusammenleben, leben würden.“
Zu den ökologischen Bedrohungen zählten Nahrungsmittelknappheit, Infektionskrankheiten, Naturkatastrophen und Krieg. In einer zweiten Studie konnte der Zusammenhang zwischen Ausmaß der Bedrohung und Vorurteilen auch für die 50 Staaten der USA nachgewiesen werden.

Vorurteile sind das Gegenteil von Offenheit. Diese jedoch braucht es bei der Lösung neuer Probleme, insbesondere, wenn sie globaler Natur sind und tatsächliche tiefgreifende Veränderungen persönlicher Präferenzen und Handlungsweisen betreffen. Unsere Tendenz, auf Bedrohungen mit Angst zu reagieren und weniger offen für Andersartigkeit und andere Menschen zu sein, mag als Reaktion auf Nahrungsmittelknappheit und Infektionskrankheiten kurzfristig funktionieren und deswegen von der Evolution gewissermaßen in unser Repertoire sozialer Notfallreaktionen eingebaut worden sein. Bei globaler Bedrohung sind diese Reaktionen jedoch kontraproduktiv. Anders ausgedrückt: Menschen reagierten zwar schon immer auf Bedrohungen, die Menschheit als Ganzes jedoch nicht. Die Evolution hat uns darauf nicht vorbereitet.

\section{Solidarität versus Entsolidarisierung}

Um den Klimawandel wirksam zu bekämpfen, müssen wir alle an einem Strang ziehen. Genau das Gegenteil geschieht jedoch derzeit auf der politischen Weltbühne: Donald Trump („America first“), Boris Johnson („Brexit“), Jarosław Kaczyński (rechtskonservative Partei „Recht und Gerechtigkeit“, PIS, in Polen) oder Viktor Orban (rechts konservativer Umbau der zuvor liberalen Partei Fidesz in Ungarn) sind deutliche Beispiele.

Schon eine Million Flüchtlinge nach Deutschland innerhalb etwa eines Jahres brachten nicht nur Deutschland (sondern auch Polen, Ungarn, Tschechien und Großbritannien) einen neuen Nationalismus und die EU an den Rand ihrer Existenz, wie die jüngste Geschichte gezeigt hat. Wenn der Klimawandel jedoch wirksam bekämpft werden soll, dann geht dies überhaupt nur als transnationale, globale Aufgabe. Keine Nation kann für sich allein den Klimawandel bekämpfen und der aufkommende Nationalismus führt zusätzlich zu mehr Konflikten und gegenseitiger Schuldzuweisung. Bei der Lösung eines globalen Problems ist die Unterscheidung von Ingroup und Outgroup definitiv nicht hilfreich².

2 Es sei hier kurz vor Drucklegung noch erwähnt, dass aus dieser Sicht alles, was zur Entsolidarisierung unser Gemeinschaft führt, selbstverständlich öffentlich kritisiert werden darf: Wenn ein Kinderchor die Großmutter als „Umweltsau“ bezeichnet, dann ist das für das wechselseitige Verständnis der Generationen ebenso wenig hilfreich, wie wenn etwa der Opa über die „verkommene Jugend“ schimpft. Die Jungen sind gesetzlich noch nicht für sich verantwortlich und es sind prinzipiell die Erwachsenen, die sie prägen (ganz gleich, ob das die unter 18-Jährigen oder die über 18-Jährigen gut finden oder nicht). Es ist faktisch und rechtlich so. Und selbst die Alten sind nicht für die Lebensumstände, die ihnen die Werte antrainiert haben, die sich heute in ihren Köpfen befinden, verantwortlich, denn diese Umstände sind vom Einzelnen praktisch so gut wie nicht und politisch nur mit einer unter Millionen Stimmen bei Wahlen zu beeinflussen. Gegenseitige Verunglimpfung und eine Vergiftung der Kommunikation zwischen den Generationen ist das Letzte, was wir gegenwärtig zur Lösung schwieriger globaler Probleme brauchen. Verantwortung für unsere Gesellschaft haben alle, und erst recht diejenigen, die als „Meinung“ getarnte vulgäre Selbstdarstellung mit öffentlichen Mitteln - also auf Kosten der Gesellschaft - verbreiten. 
Ich habe an anderer Stelle schon dargestellt, dass es zur dunklen Seite des „Kuschelhormons“ Oxytocin gehört, dass es die Unterscheidung von Menschen in Ingroup und Outgroup fördert [10]. Wir brauchen also Gemeinschaft und Solidarität, aber kein Kuscheln! Mit Immanuel Kant, dem Philosophen der Aufklärung, könnte man sagen, dass unsere Solidarität eine rational gebotene sein sollte und nicht eine emotional gefühlte. Die Gleichheit der Menschen gilt - egal, ob ich den anderen mag oder nicht. Dass Oxytocin Vorurteile gegenüber den Mitgliedern der Outgroup nachweislich verstärkt, macht die grundlegende gesellschaftliche Bedeutung dieses Gedankens nochmals sehr deutlich.

\section{Radikalisierung versus Freiheit}

„Was ist Ihnen lieber, Demokratie oder eine wirksame Einschränkung der Erderwärmung? “3 [6] - Als ich diese Frage im September 2019 zum ersten Mal las - nicht irgendwo, sondern in einem im Fachblatt New Scientist erschienenen Kommentar - war ich tief erschrocken. Wie kommt man auf so etwas? Im Grunde ist der Gedanke nicht sehr kompliziert. In der Politik wird viel geredet und wenig getan. Das liegt auch daran, dass viele Maßnahmen zur Rettung der Erde extrem unpopulär sind. Freiheiten, die wir für selbstverständlich halten, werden eingeschränkt werden müssen. Die Fragen, wie wir wirtschaften, und ob Rendite das einzige Kriterium zur Beurteilung der Güte einer Entscheidung bleiben darf, woher wir Energie und Nahrungsmittel beziehen und wie wir damit umgehen, oder wie wir unsere Infrastruktur umbauen, um sie klimafreundlicher zu gestalten, sind letztlich alle politisch.

Was soll geschehen, wenn sich herausstellt, dass es in keiner Demokratie dieser Welt möglich ist, genügend Menschen zu überzeugen, die notwendigen Opfer zu bringen, um eine Umweltkatastrophe abzuwenden? Wenn also die Mehrheit der Menschen eher kurzfristig und egoistisch denkt? Demokratie lebt ja davon, dass jeder seine Eigeninteressen vertritt und genau dafür stimmt. Es ist nicht auszuschließen, dass sich herausstellt, dass die Interessen der Leute, die in 100 Jahren auf der Erde leben, heute von niemandem vertreten werden. Hand aufs Herz: Im Moment sieht es nicht danach aus, dass diese Überlegungen rein hypothetischer Natur sind!

Von hier ist es nur noch ein kleiner Schritt zu folgender Aussage: „Some may look with envy to authoritarian regimes such as China, which has stated its aim to build an "ecological civilisation" and, politically, looks more capable of doing so than any democracy. " - Kein Wunder, dass Der Spiegel in einem Leitartikel nur eine Woche nach dem Kommentar im New Scientist von einer mit allen Mitteln abzuwendenden „Klimadiktatur“ sprach [5]. Was aber geschieht, wenn manchen irgendwann wirklich und ganz wörtlich das Wasser bis zum Hals steht? Und wie viele Jahre oder Jahrzehnte vorher wird es nicht nur zu harmlosem Flugschämen, sondern zur öffentlichen Ächtung von Rindfleisch, SUVs, Kreuzfahrten und Überseereisen kommen? „Der Klimawandel wird zeigen, wie gut die Demokratie als Herrschaftsform ist. " Man hört diesen Satz mittlerweile immer öfter. Vielleicht ist er ja optimistisch gemeint: Der

3 Im Original: "At some point, then, environmentalists may have to ask themselves a very difficult question: which is more important, saving the planet or saving democracy?"
Klimawandel wird die Demokratie und damit die Menschen besser machen müssen, wenn diese eine Chance haben wollen.

Vielleicht sei an dieser Stelle doch noch ein kleiner Tipp verraten, nicht für Politiker, sondern für die Medienmacher: Solange in den Nachrichten von einer „schwächelnden“ Konjunktur bei der Luftfahrt die Rede ist, wird deutlich, dass wir die Ökonomie für wichtiger halten als die Ökologie, denn wir könnten im Hinblick auf die gleichen Daten auch von einer „Verbesserung“ der Chancen der Erde reden. Solange wir in den gleichen Nachrichten sogar die ökonomisch „schwächelnde Luftfahrt“ und - eine Minute später - die „hohen Treibhausgase durch die Luftfahrt“ als Hiobsbotschaften dargeboten bekommen, wird klar, dass es in den Nachrichten nicht um die Lösung von Problemen geht, sondern darum, die Leute mit schlechten Nachrichten zu erschrecken, weil man damit mehr Zuschauer hat. Das wiederum liegt am Geschäftsmodell der Medien: Die Quoten müssen stimmen. Und mit Angst und Schrecken geht das besser als mit Nachdenken!

Womit wir wieder bei meinem ersten Punkt wären: „Angst essen Seele auf" 4 - Aber nicht nur, was deren Kreativität anbelangt. Es geht vielmehr auch um Solidarität, Freiheit, Gleichheit und Wahrheit - die guten alten Werte der Aufklärung. An diese zu erinnern erscheint mir gerade heute wichtiger denn je.

\section{Literatur}

[1] APA, OPP International Summit on Psychology and Global Health. A Leader in Climate Action. Lissabon, 15.11.2019 www.psychologyandglobalhealth.org/; abgerufen am 6.1.2010

[2] Fears R, ter Meulen V, von Braun J. Global food and nutrition security needs more and new science. Science Advances 2019; 5: eaba2946

[3] Hill AC, Martinez-Diaz L. Building a Resilient Tomorrow. Oxford: University Press; 2019

[4] Jackson JC, van Egmond M, Choi VK, et al. Ecological and cultural factors underlying the global distribution of prejudice. PLoS ONE 2019; 14: e0221953. doi.org/10.1371/journal.pone.0221953

[5] Kurbjuweit D. Freiheit kontra Wirksamkeit. Die neuen Klimagesetze werden zeigen, wie gut unsere Demokratie ist. DER SPIEGEL 20.9.2019.

[6] Lawton G. Brexit makes us question democracy - and so does climate change. New Scientist 2019; 3247. www.newscientist.com/article/ mg24332472-400-brexit-makes-us-question-democracy-and-so-doesclimate-change/

[7] Schmidt C. An even bigger climate problem. Science 2019; 366: 1053

[8] Sörqvist P, Marsh JE (Hrsg.). The Cognitive Psychology of Climate Change. Lausanne: Frontiers Media; 2019.

[9] Spitzer M. Oxytocin - die dunkle Seite des Kuschelhormons. Nervenheilkunde 2012; 31: 653-656

[10] Spitzer M: Biologie versus Kultur, Sprache und Denken - am Beispiel von Farben und Emotionen. Nervenheilkunde 2020; 39: 75-82

[11] Van der Linden S, Maibach E, Leiserowitz A. Improving Public Engagement with Climate Change: Five "Best Practice". Insights From Psychological Science. Perspectives on Psychological Science 2015; 10: 758-763

[12] Vaughan A. Climate Summit Failure. New Scientist 2019; 244 (3261/3262): 5

[13] Spitzer M. Christiania, Science und Cocktails. Nervenheilkunde 2019; 38: $453-458$

4 So lautet der Titel eines Films des deutschen Regisseurs Rainer Werner Fassbinder aus dem Jahr 1974. 